\title{
Observations and Study of Byurakan-IRAS Galaxies: Summary
}

\author{
G. A. Mikayelyan, A. M. Mickaelian, H. V. Abrahamyan, G. M. Paronyan, and M. V. \\ Gyulzadyan
}

NAS RA V. Ambartsumian Byurakan Astrophysical Observatory, Armenia

\begin{abstract}
The paper is a summary and general analysis of optical spectroscopic data on 257 ByurakanIRAS Galaxies (BIG objects) obtained with the BAO 2.6-m, SAO 6-m, OHP 1.93-m telescopes and taken from SDSS spectroscopic database. 149 star-formation regions galaxies, 42 galaxies with active nuclei, and 28 galaxies with a composite spectrum were identified. The spectra of 21 galaxies show signs of emission, but without the possibility of more precise determination of their activity type, 13 galaxies appear to have star formation rates that do not exceed normal, and 3 are absorption galaxies.
\end{abstract}

Keywords: galaxies: spectra: active galactic nuclei: starburst galaxies

\section{Introduction}

The InfraRed Astronomical Satellite (IRAS) was the first space-based observatory that performed a survey of the entire sky at infrared (IR) wavelengths. It mapped $96 \%$ of the sky in four bands: $12,25,60$, and $100 \mu \mathrm{m}$. IRAS point sources are published in two large catalogues, IRAS Point Source Catalog (PSC; IRAS 1988) and IRAS Faint Source Catalog (Moshir et al., 1990). Though FSC contains fainter sources and is deeper, however, it misses the Milky Way area $\mathrm{b}<\left|10^{\circ}\right|$. In 2015, Abrahamyan et al. (2015) cross-correlated these two catalogues and the Combined Catalogue of IRAS point sources was published containing 345,163 sources. However, many of these sources are still left without any identification, that is, their physical nature remains unclear. Thus, the question of identifying these objects in the visible and obtaining optical spectra for classifying them has arisen. This may lead to a revision of previous ideas regarding the relative distribution of various types of galaxies. About 75,000 of them are believed to be starburst (SB) galaxies, still undergoing their star-formation phase. IRAS sources have increased our understanding of objects in Our Galaxy (stars and nebulae), especially star formation processes in galaxies, activity of galactic nuclei, and galaxy interactions. In particular, there is a special interest in the discovery of galaxies with high IR luminosity: LIRG (Luminous InfraRed Galaxies), ULIRG (Ultra-Luminous InfraRed Galaxies), and HLIRG (Hyper-Luminous InfraRed Galaxies) (Sanders and Mirabel, 1996). Latest IR surveys have shown that ULIRGs are vastly more numerous at high redshifts. Understanding the physics and evolution of ULIRGs, the contribution of high redshift ULIRGs to the cosmic IR background and the global history of star formation, and the role of ULIRGs as diagnostics of the formation of massive galaxies and large-scale structures are important questions still to be clarified. Though a number of recent IR (especially near-IR and mid-IR) surveys appeared: 2MASS (Cutri et al., 2003, Skrutskie et al., 2006); AllWISE (Cutri et al., 2013); AKARI (Ishihara et al., 2010, Yamamura et al., 2010), IRAS catalogues are still useful for studies at far-IR (FIR) wavelengths (60 and $100 \mu \mathrm{m})$, i.e., for extragalactic studies, and IRAS galaxies provide homogeneous samples of IR-selected AGN and SB.

There have been a number of studies on identifications of IRAS galaxies since the release of IRAS catalogs: IRAS Revised Bright Galaxy Sample (Sanders et al., 2003); Far-InfraRed (FIR) sources

*gormick@mail.ru, Corresponding author 
(Bertin et al., 1997); IRAS galaxies towards the Boötes void (Strauss and Huchra, 1988); IRAS point sources in the area of Fornax, Hydra I and Coma clusters (Wang et al., 1991); IRAS $1.2 \mu \mathrm{m}$ survey (Fisher et al., 1995); IRAS galaxies in Virgo cluster area (Yuan et al., 1996); and some others.

About half of all IRAS sources are still not identified and there is a need for optical identifications. Since 1995, a project of optical identifications has been carried out in the Byurakan Astrophysical Observatory (Mickaelian, 1995), in order to detect new galaxies with bursts of star formation in their central regions (SB, or Starburst galaxies) (Weedman et al., 1981), galaxies with active nuclei (AGN, active galactic nuclei) (Ambartsumian, 1958), interacting pairs, and galaxies with high IR luminosity (ULIRG, Ultra-Luminous IR Galaxies), which resulted in revealing 1178 galaxies and 399 stars, named Byurakan-IRAS Galaxies (BIG) (Mickaelian and Sargsyan, 2004) and Byurakan-IRAS Stars (BIS) (Mickaelian and Gigoyan, 2006), respectively. Identifications using low-dispersion spectra of the First Byurakan Survey (FBS or Markarian survey) (Markarian et al., 1989) and its digitized version, DFBS (Mickaelian et al., 2007, Massaro et al., 2008) guaranteed better selection of optical counterparts compared to other identification works.

BIG objects have been studied spectroscopically using BAO $2.6 \mathrm{~m}$ (Mickaelian et al., 2003, Sargsyan and Mickaelian, 2006), Special Astrophysical Observatory (SAO, Russia) 6 m (Mickaelian et al., 1998, Balayan et al., 2001), Observatoire de Haute-Provence (OHP, France) 1.93 m (Mickaelian, 2004) telescopes and the Sloan Digital Sky Survey (Abolfathi et al., 2018) (Mickaelian et al., 2018). Altogether 255 BIG objects have been studied and classified. The spectroscopic studies of BIG objects facilitate the concurrent solution of several problems. These problems range from confirming the extragalactic nature of objects and determining their redshifts to detailed analyses of the objects' structure, which proved to be of greatest interest, such as galaxies with enhanced IR luminosities and/or with nuclear or starburst activity.

\section{BIG Sample}

A total of 257 spectra were obtained: 56 with the BAO telescope, 54 with the SAO telescope, 64 with the OHP telescope and 83 from the SDSS. The list of all objects with their parameters are given in Table 1. The columns list spectra source (telescope name or SDSS), the IRAS names for the objects, stellar magnitudes close to $V$ (for objects from SDSS the $r$ band was taken), the radial velocities $\left(v_{r}\right)$ determined from the emission $z$ (and calculated using the relativistic formula), the corresponding distances of the objects calculated for $H=71 \mathrm{~km} /(s \times M p c)$, the absolute stellar magnitudes $(M)$, the redshifts determined from the emission lines $\left(z_{e m}\right)$, and the type of activity of the objects (":" denotes objects with uncertain classifications).

Table 1: The list of all observed BIG objects with their parameters

\begin{tabular}{|l|c|c|c|c|c|c|c|}
\hline IRAS source & $\mathbf{m}_{V}$ & Spectra source & $\mathbf{z}_{e m}$ & $\mathbf{v}_{r}(\mathrm{~km} / \mathrm{s})$ & $\mathbf{R}(\mathrm{Mpc})$ & $\mathbf{M}$ & Activity type \\
\hline $03304+8456 \mathrm{a}$ & 16.8 & OHP $1.93 \mathrm{~m}$ & 0.0509 & 14868 & 209 & -19.80 & HII \\
$03304+8456 \mathrm{~b}$ & 16.4 & BAO $2.6 \mathrm{~m}$ & 0.0500 & 14625 & 206 & -20.16 & Abs \\
$03333+7851$ & 15.0 & OHP $1.93 \mathrm{~m}$ & 0.0561 & 16347 & 230 & -21.81 & HII \\
$03347+7748$ & 15.5 & OHP $1.93 \mathrm{~m}$ & 0.0346 & 10209 & 144 & -20.29 & Composite \\
$03386+7909$ & 15.9 & OHP $1.93 \mathrm{~m}$ & 0.0559 & 16313 & 230 & -20.91 & Sy 2 \\
$03424+8424$ & 16.0 & SAO $6 \mathrm{~m}$ & 0.0744 & 21492 & 303 & -21.41 & LINER: \\
$03424+8713$ & 15.4 & BAO $2.6 \mathrm{~m}$ & 0.0249 & 7365 & 104 & -19.67 & HII \\
$03485+7703$ & 15.6 & OHP $1.93 \mathrm{~m}$ & 0.0701 & 20281 & 286 & -21.68 & HII \\
$04033+6942$ & 15.9 & OHP $1.93 \mathrm{~m}$ & 0.0161 & 4803 & 68 & -18.25 & LINER \\
$04079+7033$ & 14.5 & OHP $1.93 \mathrm{~m}$ & 0.0132 & 3934 & 55 & -19.22 & HII \\
$04140+7448$ & 16.0 & OHP $1.93 \mathrm{~m}$ & 0.0338 & 9972 & 140 & -19.74 & AGN \\
$04183+7457$ & 14.7 & OHP $1.93 \mathrm{~m}$ & 0.0325 & 9589 & 135 & -20.95 & Composite \\
$04574+7639$ & 17.1 & BAO $2.6 \mathrm{~m}$ & 0.1209 & 34081 & 480 & -21.29 & Em \\
$05097+7954$ & 15.2 & OHP $1.93 \mathrm{~m}$ & 0.0570 & 16602 & 234 & -21.64 & HII \\
\hline
\end{tabular}


Table 1 - continued from previous page

\begin{tabular}{|c|c|c|c|c|c|c|c|}
\hline IRAS source & $\mathbf{m}_{V}$ & Spectra source & $\mathbf{z}_{e m}$ & $\mathbf{v}_{r}(\mathrm{~km} / \mathrm{s})$ & $\mathbf{R}$ (Mpc) & $\mathbf{M}$ & Activity type \\
\hline $05126+6516$ & 15.4 & OHP $1.93 \mathrm{~m}$ & 0.0402 & 11824 & 167 & -20.71 & HII \\
\hline $05196+7257$ & 17.4 & OHP $1.93 \mathrm{~m}$ & 0.1030 & 29303 & 413 & -20.68 & Sy2 \\
\hline $05214+7741$ & 16.3 & OHP $1.93 \mathrm{~m}$ & 0.0769 & 22194 & 313 & -21.17 & AGN \\
\hline $05229+6826$ & 14.5 & OHP $1.93 \mathrm{~m}$ & 0.0166 & 4950 & 70 & -19.72 & HII \\
\hline $05275+6600$ & 16.0 & OHP $1.93 \mathrm{~m}$ & 0.0306 & 9037 & 127 & -19.52 & AGN: \\
\hline $05395+7550$ & 15.0 & OHP $1.93 \mathrm{~m}$ & 0.0248 & 7342 & 103 & -20.07 & HII \\
\hline $05401+6435$ & 15.2 & SAO $6 \mathrm{~m}$ & 0.0539 & 15735 & 222 & -21.53 & HII \\
\hline $05475+7449$ & 16.0 & OHP $1.93 \mathrm{~m}$ & 0.0515 & 15061 & 212 & -20.63 & Composite \\
\hline $05577+6141$ & 16.1 & OHP $1.93 \mathrm{~m}$ & 0.0306 & 9034 & 127 & -19.42 & Em \\
\hline $06022+7559$ & 16.8 & OHP $1.93 \mathrm{~m}$ & 0.0817 & 23509 & 331 & -20.80 & HII \\
\hline $06028+6734 a$ & 15.9 & BAO $2.6 \mathrm{~m}$ & 0.0169 & 5015 & 71 & -18.33 & HII \\
\hline $06028+6734 b$ & 15.9 & BAO $2.6 \mathrm{~m}$ & 0.0168 & 4983 & 70 & -18.31 & HII \\
\hline $06038+6239$ & 14.4 & $\mathrm{SAO} 6 \mathrm{~m}$ & 0.0413 & 12134 & 171 & -21.76 & Norm. \\
\hline $06261+7818 \mathrm{a}$ & 18.4 & BAO $2.6 \mathrm{~m}$ & 0.0939 & 26855 & 378 & -19.45 & HII \\
\hline $06261+7818 b$ & 17.4 & BAO $2.6 \mathrm{~m}$ & 0.0940 & 26883 & 379 & -20.48 & LINER: \\
\hline $06273+6858 \mathrm{a}$ & 17.2 & BAO $2.6 \mathrm{~m}$ & 0.0664 & 19252 & 271 & -19.96 & Em \\
\hline $06273+6858 b$ & 16.4 & OHP 1.93m & 0.0665 & 19299 & 272 & -20.77 & HII \\
\hline $06273+6858 \mathrm{c}$ & 15.7 & OHP $1.93 \mathrm{~m}$ & 0.0656 & 19022 & 268 & -21.44 & Sy2 \\
\hline $06319+7536$ & 18.1 & OHP $1.93 \mathrm{~m}$ & 0.0915 & 26210 & 369 & -19.74 & Composite \\
\hline $06432+8551$ & 19.0 & BAO $2.6 \mathrm{~m}$ & 0.0898 & 25743 & 363 & -18.80 & HII \\
\hline $06432+8551 b$ & 16.5 & $\mathrm{SAO} 6 \mathrm{~m}$ & 0.0891 & 25544 & 360 & -21.28 & HII \\
\hline $06545+6647$ & 16.1 & OHP $1.93 \mathrm{~m}$ & 0.0160 & 4765 & 67 & -18.03 & HII \\
\hline $06584+6716 a$ & 17.0 & BAO $2.6 \mathrm{~m}$ & 0.0734 & 21214 & 299 & -20.38 & Composite \\
\hline $06584+6716 b$ & 17.9 & BAO $2.6 \mathrm{~m}$ & 0.0710 & 20546 & 289 & -19.41 & Unknown \\
\hline $07007+8242$ & 16.9 & $\mathrm{SAO} 6 \mathrm{~m}$ & 0.0586 & 17066 & 240 & -20.00 & HII \\
\hline $07021+7349$ & 14.0 & OHP $1.93 \mathrm{~m}$ & 0.1031 & 29335 & 413 & -24.08 & LINER: \\
\hline $07158+7706$ & 15.5 & OHP $1.93 \mathrm{~m}$ & 0.0485 & 14198 & 200 & -21.00 & HII \\
\hline $07205+7842$ & 16.3 & OHP 1.93m & 0.0866 & 24859 & 350 & -21.42 & Sy2 \\
\hline $07225+7653$ & 16.1 & OHP $1.93 \mathrm{~m}$ & 0.0484 & 14160 & 199 & -20.40 & AGN \\
\hline $07479+7832$ & 18.3 & OHP 1.93m & 0.1734 & 47577 & 670 & -20.83 & Em \\
\hline $08036+7211$ & 15.4 & BAO $2.6 \mathrm{~m}$ & 0.0387 & 11374 & 160 & -20.63 & Em \\
\hline $08054+6824$ & 14.1 & OHP $1.93 \mathrm{~m}$ & 0.0413 & 12123 & 171 & -22.06 & Composite \\
\hline $08095+6445$ & 14.1 & $\mathrm{SAO} 6 \mathrm{~m}$ & 0.0294 & 8690 & 122 & -21.34 & Norm. \\
\hline $08142+6821$ & 16.0 & OHP $1.93 \mathrm{~m}$ & 0.0388 & 11414 & 161 & -20.03 & HII \\
\hline $08247+7311$ & 14.9 & OHP 1.93m & 0.0655 & 19013 & 268 & -22.24 & HII \\
\hline $08259+7427$ & 17.3 & OHP 1.93m & 0.1232 & 34685 & 489 & -21.14 & HII \\
\hline $08303+6118$ & 16.8 & SDSS & 0.0865 & 24831 & 350 & -20.89 & HII \\
\hline $08317+7602$ & 17.0 & OHP $1.93 \mathrm{~m}$ & 0.0931 & 26632 & 375 & -20.87 & HII \\
\hline $08339+6517$ & 14.4 & BAO $2.6 \mathrm{~m}$ & 0.0190 & 5634 & 79 & -20.12 & HII \\
\hline $08379+6753$ & 16.0 & BAO $2.6 \mathrm{~m}$ & 0.0363 & 10687 & 151 & -19.89 & HII \\
\hline $08410+6124$ & 16.3 & SDSS & 0.0769 & 22189 & 313 & -21.18 & HII \\
\hline $08567+6325$ & 15.7 & SDSS & 0.0386 & 11347 & 160 & -20.31 & HII/Sy2 \\
\hline $09002+8106$ & 16.6 & $\mathrm{SAO} 6 \mathrm{~m}$ & 0.0494 & 14454 & 204 & -19.94 & HII \\
\hline $09020+6751 \mathrm{a}$ & 16.7 & BAO $2.6 \mathrm{~m}$ & 0.0505 & 14754 & 208 & -19.89 & LINER \\
\hline $09020+6751 b$ & 16.1 & BAO $2.6 \mathrm{~m}$ & 0.0564 & 16435 & 231 & -20.74 & Em \\
\hline $09037+6937$ & 16.8 & OHP 1.93m & 0.0125 & 3738 & 53 & -16.81 & HII \\
\hline $09056+6538$ & 16.3 & BAO $2.6 \mathrm{~m}$ & 0.0652 & 18910 & 266 & -20.88 & Composite \\
\hline $09056+6538$ & 16.3 & SDSS & 0.0649 & 18829 & 265 & -20.80 & HII \\
\hline $09062+8134 a$ & 16.8 & $\mathrm{SAO} 6 \mathrm{~m}$ & 0.0495 & 14483 & 204 & -19.75 & LINER: \\
\hline $09062+8134 b$ & 17.9 & $\mathrm{SAO} 6 \mathrm{~m}$ & 0.0491 & 14369 & 202 & -18.63 & HII \\
\hline $09103+8326$ & 17.1 & $\mathrm{SAO} 6 \mathrm{~m}$ & 0.0497 & 14540 & 205 & -19.46 & HII \\
\hline
\end{tabular}


Table 1 - continued from previous page

\begin{tabular}{|c|c|c|c|c|c|c|c|}
\hline IRAS source & $\mathbf{m}_{V}$ & Spectra source & $\mathbf{z}_{e m}$ & $\mathbf{v}_{r}(\mathrm{~km} / \mathrm{s})$ & $\mathbf{R}$ (Mpc) & $\mathbf{M}$ & Activity type \\
\hline $09162+6539$ & 14.6 & SDSS & 0.0379 & 11143 & 157 & -21.35 & HII \\
\hline $09162+6539$ & 15.5 & OHP $1.93 \mathrm{~m}$ & 0.0379 & 11160 & 157 & -20.48 & HII \\
\hline $09173+6231$ & 15.6 & SDSS & 0.0473 & 13859 & 195 & -20.90 & HII \\
\hline $09176+6544$ & 15.2 & SDSS & 0.0190 & 5640 & 79 & -19.26 & HII \\
\hline $09180+6532$ & 16.0 & OHP $1.93 \mathrm{~m}$ & 0.0382 & 11230 & 158 & -20.00 & HII \\
\hline $09229+7731$ & 15.0 & BAO $2.6 \mathrm{~m}$ & 0.0092 & 2741 & 39 & -17.93 & HII \\
\hline $09233+7825$ & 14.7 & OHP $1.93 \mathrm{~m}$ & 0.0747 & 21586 & 304 & -22.71 & Em \\
\hline $09244+7405$ & 17.2 & OHP $1.93 \mathrm{~m}$ & 0.1586 & 43847 & 618 & -21.75 & Sy2 \\
\hline $09273+6232$ & 16.3 & SDSS & 0.0420 & 12350 & 174 & -19.89 & AGN: \\
\hline $09305+6813$ & 15.7 & OHP $1.93 \mathrm{~m}$ & 0.0710 & 20548 & 289 & -21.61 & Sy2: \\
\hline $09343+6450$ & 15.8 & SDSS & 0.0710 & 20542 & 289 & -21.53 & Sy2: \\
\hline $09363+6655$ & 15.9 & SDSS & 0.0596 & 17355 & 244 & -21.04 & HII \\
\hline $09386+6240$ & 15.6 & SDSS & 0.0397 & 11661 & 164 & -20.48 & Em \\
\hline $09406+6840$ & 15.6 & SDSS & 0.0118 & 3506 & 49 & -17.89 & HII \\
\hline $09406+6840$ & 16.3 & OHP $1.93 \mathrm{~m}$ & 0.0119 & 3561 & 50 & -17.20 & HII \\
\hline $09418+8124$ & 16.0 & $\mathrm{SAO} 6 \mathrm{~m}$ & 0.0398 & 11703 & 165 & -20.09 & Abs \\
\hline $09427+7528$ & 17.6 & BAO $2.6 \mathrm{~m}$ & 0.1099 & 31168 & 439 & -20.61 & HII \\
\hline $09477+7050 a$ & 18.5 & $\mathrm{SAO} 6 \mathrm{~m}$ & 0.1268 & 35645 & 502 & -20.00 & Norm. \\
\hline $09477+7050 \mathrm{~b}$ & 18.0 & $\mathrm{SAO} 6 \mathrm{~m}$ & 0.1270 & 35698 & 503 & -20.51 & HII \\
\hline $09477+7050 c$ & 17.5 & $\mathrm{SAO} 6 \mathrm{~m}$ & 0.1277 & 35881 & 505 & -21.02 & HII \\
\hline $09571+8435$ & 16.5 & $\mathrm{SAO} 6 \mathrm{~m}$ & 0.0924 & 26444 & 372 & -21.36 & HII \\
\hline $10045+7502 b$ & 16.0 & BAO $2.6 \mathrm{~m}$ & 0.0481 & 14083 & 198 & -20.49 & LINER \\
\hline $10172+7548$ & 16.2 & OHP $1.93 \mathrm{~m}$ & 0.0592 & 17235 & 243 & -20.73 & HII \\
\hline $10210+7528$ & 15.5 & OHP $1.93 \mathrm{~m}$ & 0.0279 & 8245 & 116 & -19.82 & HII \\
\hline $10228+6227$ & 17.2 & SDSS & 0.1163 & 32871 & 463 & -21.17 & HII \\
\hline $10252+7013$ & 16.0 & OHP $1.93 \mathrm{~m}$ & 0.1180 & 33319 & 469 & -22.36 & HII \\
\hline $10270+7302$ & 12.5 & OHP $1.93 \mathrm{~m}$ & 0.0224 & 6654 & 94 & -22.36 & HII \\
\hline $10272+6953$ & 14.5 & OHP $1.93 \mathrm{~m}$ & 0.0387 & 11391 & 160 & -21.53 & Composite \\
\hline $10276+7443$ & 17.3 & OHP $1.93 \mathrm{~m}$ & 0.0576 & 16769 & 236 & -19.57 & AGN \\
\hline $10298+6119$ & 15.2 & SDSS & 0.0293 & 8666 & 122 & -20.22 & HII \\
\hline $10331+6338$ & 15.9 & SDSS & 0.0381 & 11202 & 158 & -20.04 & HII \\
\hline $10361+7952 \mathrm{a}$ & 15.9 & BAO $2.6 \mathrm{~m}$ & 0.0388 & 11414 & 161 & -20.16 & HII \\
\hline $10361+7952 b$ & 15.5 & BAO $2.6 \mathrm{~m}$ & 0.0394 & 11587 & 163 & -20.56 & HII/Comp: \\
\hline $10383+7637$ & 16.2 & OHP $1.93 \mathrm{~m}$ & 0.0315 & 9293 & 131 & -19.38 & AGN \\
\hline $10486+6558$ & 16.7 & SDSS & 0.0338 & 9960 & 140 & -18.99 & HII \\
\hline $10527+7136$ & 16.4 & BAO $2.6 \mathrm{~m}$ & 0.1011 & 28790 & 405 & -21.68 & Composite \\
\hline $10529+7144$ & 14.0 & OHP $1.93 \mathrm{~m}$ & 0.0619 & 17996 & 253 & -23.02 & HII \\
\hline $10541+6614$ & 17.3 & SDSS & 0.1306 & 36641 & 516 & -21.22 & $\mathrm{Em}$ \\
\hline $10589+6515$ & 16.1 & SDSS & 0.0775 & 22362 & 315 & -21.40 & Sy2 \\
\hline $11008+7915 a$ & 16.7 & OHP 1.93m & 0.0620 & 18013 & 254 & -20.32 & HII \\
\hline $11008+7915 b$ & 16.5 & OHP $1.93 \mathrm{~m}$ & 0.0620 & 18013 & 254 & -20.52 & HII \\
\hline $11053+7037$ & 14.0 & OHP $1.93 \mathrm{~m}$ & 0.0412 & 12097 & 170 & -22.16 & HII \\
\hline $11059+7117$ & 17.9 & OHP $1.93 \mathrm{~m}$ & 0.1388 & 38761 & 546 & -20.79 & Composite \\
\hline $11067+7024 a$ & 15.5 & BAO $2.6 \mathrm{~m}$ & 0.0389 & 11443 & 161 & -20.54 & HII/Comp: \\
\hline $11067+7024 b$ & 16.0 & BAO $2.6 \mathrm{~m}$ & 0.0389 & 11443 & 161 & -20.04 & HII \\
\hline $11069+7438$ & 16.2 & OHP $1.93 \mathrm{~m}$ & 0.0395 & 11602 & 163 & -19.87 & HII \\
\hline $11085+7712 \mathrm{a}$ & 15.6 & OHP 1.93m & 0.1003 & 28574 & 402 & -22.42 & Composite \\
\hline $11085+7712 b$ & 18.0 & OHP $1.93 \mathrm{~m}$ & 0.0996 & 28404 & 400 & -20.01 & AGN \\
\hline $11161+6629$ & 15.5 & SDSS & 0.0431 & 12663 & 178 & -20.78 & HII \\
\hline $11201+6305$ & 16.9 & SDSS & 0.1905 & 51799 & 730 & -22.41 & Em \\
\hline $11201+6305$ & 15.7 & SDSS & 0.0111 & 3311 & 47 & -17.66 & HII \\
\hline
\end{tabular}


Table 1 - continued from previous page

\begin{tabular}{|c|c|c|c|c|c|c|c|}
\hline IRAS source & $\mathbf{m}_{V}$ & Spectra source & $\mathbf{z}_{e m}$ & $\mathbf{v}_{r}(\mathrm{~km} / \mathrm{s})$ & $\mathbf{R}_{(\mathrm{Mpc})}$ & $\mathbf{M}$ & Activity type \\
\hline $11371+8106$ & 16.7 & BAO $2.6 \mathrm{~m}$ & 0.0445 & 13053 & 184 & -19.60 & HII \\
\hline $11401+6642$ & 15.4 & SDSS & 0.0554 & 16169 & 228 & -21.36 & HII \\
\hline $11436+7438$ & 14.8 & OHP $1.93 \mathrm{~m}$ & 0.0560 & 16333 & 230 & -22.01 & Composite \\
\hline $11462+6424$ & 15.3 & SDSS & 0.0414 & 12169 & 171 & -20.87 & HII \\
\hline $11472+6647$ & 15.3 & SDSS & 0.0402 & 11809 & 166 & -20.78 & Sy2 \\
\hline $11497+6139$ & 16.0 & SDSS & 0.0427 & 12530 & 176 & -20.21 & HII \\
\hline $11587+6124$ & 17.4 & SDSS & 0.1638 & 45166 & 636 & -21.66 & HII \\
\hline $12008+6141$ & 15.6 & SDSS & 0.0644 & 18689 & 263 & -21.48 & HII/LINER \\
\hline $12040+8158$ & 17.7 & BAO $2.6 \mathrm{~m}$ & 0.0903 & 25858 & 364 & -20.14 & HII \\
\hline $12069+6753$ & 17.1 & SDSS & 0.0585 & 17026 & 240 & -19.83 & HII \\
\hline $12077+8131$ & 16.5 & BAO $2.6 \mathrm{~m}$ & 0.0397 & 11671 & 164 & -19.56 & HII \\
\hline $12119+6117$ & 17.3 & SDSS & 0.1630 & 44948 & 633 & -21.72 & Em \\
\hline $12120+6838$ & 15.5 & SDSS & 0.0602 & 17512 & 247 & -21.48 & HII \\
\hline $12120+6838$ & 15.5 & SDSS & 0.0606 & 17643 & 248 & -21.44 & Sy1.9 \\
\hline $12120+6838 a$ & 15.8 & BAO $2.6 \mathrm{~m}$ & 0.0581 & 16924 & 238 & -21.09 & HII \\
\hline $12120+6838 b$ & 16.2 & BAO $2.6 \mathrm{~m}$ & 0.0604 & 17574 & 248 & -20.77 & HII \\
\hline $12120+6838 c$ & 15.6 & BAO $2.6 \mathrm{~m}$ & 0.0597 & 17376 & 245 & -21.34 & Composite \\
\hline $12138+7537 a$ & 16.9 & BAO $2.6 \mathrm{~m}$ & 0.0497 & 14540 & 205 & -19.67 & HII \\
\hline $12138+7537 b$ & 15.5 & BAO $2.6 \mathrm{~m}$ & 0.0468 & 13712 & 193 & -20.93 & Em \\
\hline $12138+7537 \mathrm{c}$ & 16.5 & BAO $2.6 \mathrm{~m}$ & 0.0525 & 15337 & 216 & -20.17 & LINER \\
\hline $12147+6306$ & 15.4 & SDSS & 0.0501 & 14656 & 206 & -21.14 & HII \\
\hline $12147+6306$ & 13.9 & $\mathrm{SAO} 6 \mathrm{~m}$ & 0.0505 & 14768 & 208 & -22.69 & Norm. \\
\hline $12164+6437$ & 15.0 & SDSS & 0.0308 & 9104 & 128 & -20.57 & HII \\
\hline $12207+6329$ & 15.0 & SDSS & 0.0589 & 17154 & 242 & -21.87 & HII \\
\hline $12226+6630$ & 17.4 & SDSS & 0.0865 & 24819 & 350 & -20.37 & Em \\
\hline $12226+6630$ & 16.4 & SDSS & 0.0899 & 25762 & 363 & -21.45 & HII \\
\hline $12235+6253$ & 16.6 & SDSS & 0.0672 & 19479 & 274 & -20.60 & AGN \\
\hline $12267+6540$ & 16.4 & SDSS & 0.0504 & 14728 & 207 & -20.15 & HII \\
\hline $12312+6939$ & 15.4 & OHP $1.93 \mathrm{~m}$ & 0.0692 & 20035 & 282 & -21.85 & HII \\
\hline $12395+6238$ & 14.2 & $\mathrm{SAO} 6 \mathrm{~m}$ & 0.0342 & 10085 & 142 & -21.56 & Composite \\
\hline $12395+6238$ & 15.7 & SDSS & 0.0338 & 9959 & 140 & -20.08 & HII \\
\hline $12470+6705$ & 17.4 & SDSS & 0.1295 & 36345 & 512 & -21.14 & HII \\
\hline $12477+7936 \mathrm{a}$ & 14.7 & OHP $1.93 \mathrm{~m}$ & 0.0338 & 9972 & 140 & -21.04 & HII \\
\hline $12483+7332$ & 14.9 & OHP $1.93 \mathrm{~m}$ & 0.0314 & 9269 & 131 & -20.68 & HII \\
\hline $12502+7625$ & 16.5 & OHP 1.93m & 0.0634 & 18424 & 259 & -20.57 & HII \\
\hline $13014+6146$ & 15.3 & SDSS & 0.0269 & 7968 & 112 & -19.93 & HII \\
\hline $13014+6146$ & 14.0 & $\mathrm{SAO} 6 \mathrm{~m}$ & 0.0272 & 8049 & 113 & -21.27 & Norm. \\
\hline $13030+6102$ & 15.4 & $\mathrm{SAO} 6 \mathrm{~m}$ & 0.0698 & 20211 & 285 & -21.87 & HII \\
\hline $13045+7016 \mathrm{a}$ & 16.2 & OHP $1.93 \mathrm{~m}$ & 0.0646 & 18752 & 264 & -20.91 & Em \\
\hline $13045+7016 b$ & 16.4 & OHP $1.93 \mathrm{~m}$ & 0.0645 & 18736 & 264 & -20.71 & HII \\
\hline $13121+6646$ & 16.0 & SDSS & 0.0677 & 19627 & 276 & -21.20 & HII \\
\hline $13209+6353$ & 17.5 & SDSS & 0.1997 & 54016 & 761 & -21.91 & Sy2 \\
\hline $13234+6239$ & 16.0 & SDSS & 0.0418 & 12272 & 173 & -20.17 & HII \\
\hline $13286+7258$ & 14.0 & OHP $1.93 \mathrm{~m}$ & 0.0305 & 8996 & 127 & -21.51 & AGN \\
\hline $13291+6524$ & 17.3 & SDSS & 0.0365 & 10738 & 151 & -18.62 & LINER: \\
\hline $13300+6652$ & 17.4 & SDSS & 0.1044 & 29694 & 418 & -20.71 & AGN \\
\hline $13300+7219$ & 15.2 & OHP $1.93 \mathrm{~m}$ & 0.0360 & 10594 & 149 & -20.67 & Composite \\
\hline $13367+6703$ & 17.2 & SDSS & 0.0933 & 26680 & 376 & -20.68 & HII \\
\hline $13386+6557$ & 16.2 & SDSS & 0.0480 & 14047 & 198 & -20.26 & HII \\
\hline $13410+7837$ & 16.0 & BAO $2.6 \mathrm{~m}$ & 0.0580 & 16905 & 238 & -20.93 & Composite \\
\hline $13503+6104$ & 16.6 & SDSS & 0.0880 & 25230 & 355 & -21.11 & HII \\
\hline
\end{tabular}


Table 1 - continued from previous page

\begin{tabular}{|c|c|c|c|c|c|c|c|}
\hline IRAS source & $\mathbf{m}_{V}$ & Spectra source & $\mathbf{z}_{e m}$ & $\mathbf{v}_{r}(\mathrm{~km} / \mathrm{s})$ & $\mathbf{R}$ (Mpc) & $\mathbf{M}$ & Activity type \\
\hline $13513+6616$ & 15.9 & SDSS & 0.0702 & 20331 & 286 & -21.38 & HII \\
\hline $13524+6213$ & 16.5 & SDSS & 0.0729 & 21076 & 297 & -20.86 & HII \\
\hline $13524+6213$ & 16.8 & SDSS & 0.0730 & 21111 & 297 & -20.53 & HII \\
\hline $14004+7445 \mathrm{a}$ & 15.9 & BAO $2.6 \mathrm{~m}$ & 0.0670 & 19422 & 274 & -21.28 & HII \\
\hline $14004+7445 b$ & 16.5 & BAO $2.6 \mathrm{~m}$ & 0.0673 & 19523 & 275 & -20.67 & Composite \\
\hline $14013+6520$ & 16.0 & SDSS & 0.0345 & 10173 & 143 & -19.74 & HII \\
\hline $14129+6111$ & 17.8 & SDSS & 0.1514 & 42024 & 592 & -21.11 & HII \\
\hline $14132+6552$ & 15.4 & SDSS & 0.0325 & 9585 & 135 & -20.22 & HII \\
\hline $14190+6432$ & 17.2 & SDSS & 0.1525 & 42288 & 596 & -21.70 & LINER \\
\hline $14196+7734$ & 16.6 & BAO $2.6 \mathrm{~m}$ & 0.0469 & 13732 & 193 & -19.87 & AGN \\
\hline $14230+6158$ & 16.4 & SDSS & 0.1112 & 31523 & 444 & -21.80 & Sy2 \\
\hline $14244+6435$ & 17.8 & SDSS & 0.1130 & 31992 & 451 & -20.49 & HII/Sy1.9 \\
\hline $14263+6116$ & 15.7 & SDSS & 0.0618 & 17967 & 253 & -21.28 & HII \\
\hline $14271+6437$ & 17.7 & SDSS & 0.1411 & 39375 & 555 & -20.98 & HII \\
\hline $14360+6129$ & 14.5 & $\mathrm{SAO} 6 \mathrm{~m}$ & 0.0521 & 15223 & 214 & -22.16 & HII \\
\hline $14370+6254$ & 16.6 & SDSS & 0.1154 & 32622 & 459 & -21.75 & HII \\
\hline $14386+6113$ & 16.0 & SDSS & 0.0934 & 26721 & 376 & -21.88 & HII \\
\hline 14418+6131A & 15.0 & SDSS & 0.0480 & 14044 & 198 & -21.53 & HII/LINER \\
\hline $14418+6131 \mathrm{~A}$ & 15.7 & SDSS & 0.0492 & 14410 & 203 & -20.88 & HII/LINER \\
\hline $14418+6131 \mathrm{~A}$ & 17.5 & SDSS & 0.0477 & 13958 & 197 & -18.93 & $\mathrm{Em}$ \\
\hline $14418+6131 \mathrm{~A}$ & 17.1 & SDSS & 0.0494 & 14458 & 204 & -19.49 & HII \\
\hline $14436+6258$ & 14.9 & SDSS & 0.0375 & 11036 & 155 & -21.07 & HII \\
\hline $14445+7003$ & 17.0 & BAO $2.6 \mathrm{~m}$ & 0.0518 & 15138 & 213 & -19.65 & Em \\
\hline $14458+7926$ & 16.5 & BAO $2.6 \mathrm{~m}$ & 0.0316 & 9330 & 131 & -19.09 & Sy2:/Comp \\
\hline $14464+6119$ & 16.9 & SDSS & 0.1364 & 38151 & 537 & -21.76 & Sy1.2 \\
\hline $14501+6212$ & 14.9 & $\mathrm{SAO} 6 \mathrm{~m}$ & 0.0429 & 12594 & 177 & -21.34 & Abs \\
\hline $14501+6212$ & 15.0 & SDSS & 0.0431 & 12666 & 178 & -21.23 & HII \\
\hline $14561+6312$ & 14.7 & $\mathrm{SAO} 6 \mathrm{~m}$ & 0.0432 & 12680 & 179 & -21.56 & Norm. \\
\hline $14561+6312$ & 15.2 & SDSS & 0.0426 & 12510 & 176 & -21.08 & Sy2 \\
\hline $14570+6339$ & 16.2 & $\mathrm{SAO} 6 \mathrm{~m}$ & 0.0475 & 13912 & 196 & -20.26 & LINER \\
\hline $14574+7641 a$ & 16.5 & BAO $2.6 \mathrm{~m}$ & 0.0446 & 13082 & 184 & -19.83 & HII \\
\hline $15356+6119 a$ & 16.4 & $\mathrm{SAO} 6 \mathrm{~m}$ & 0.0886 & 25407 & 358 & -21.37 & HII \\
\hline $15356+6119 b$ & 14.4 & $\mathrm{SAO} 6 \mathrm{~m}$ & 0.0882 & 25297 & 356 & -23.36 & HII \\
\hline $15374+6822$ & 15.7 & BAO $2.6 \mathrm{~m}$ & 0.0578 & 16851 & 237 & -21.17 & HII \\
\hline $15427+6141$ & 17.6 & $\mathrm{SAO} 6 \mathrm{~m}$ & 0.0573 & 16698 & 235 & -19.26 & Norm. \\
\hline $15449+6459$ & 15.3 & $\mathrm{SAO} 6 \mathrm{~m}$ & 0.0373 & 10981 & 155 & -20.65 & Norm. \\
\hline $16030+6312 \mathrm{a}$ & 16.8 & $\mathrm{SAO} 6 \mathrm{~m}$ & 0.0548 & 15990 & 225 & -19.96 & Norm. \\
\hline $16030+6312 b$ & 15.9 & $\mathrm{SAO} 6 \mathrm{~m}$ & 0.0569 & 16585 & 234 & -20.94 & HII \\
\hline $16044+6727$ & 16.1 & BAO $2.6 \mathrm{~m}$ & 0.0233 & 6906 & 97 & -18.85 & HII \\
\hline $16049+8802$ & 16.8 & BAO $2.6 \mathrm{~m}$ & 0.0541 & 15803 & 223 & -19.95 & Sy2 \\
\hline $16101+6345$ & 16.5 & $\mathrm{SAO} 6 \mathrm{~m}$ & 0.0563 & 16415 & 231 & -20.32 & HII \\
\hline $16118+6231$ & 13.3 & $\mathrm{SAO} 6 \mathrm{~m}$ & 0.0321 & 9476 & 133 & -22.33 & Composite \\
\hline $16119+8551$ & 17.1 & BAO $2.6 \mathrm{~m}$ & 0.0847 & 24335 & 343 & -20.54 & HII \\
\hline $16358+6709 a$ & 15.0 & BAO $2.6 \mathrm{~m}$ & 0.0534 & 15593 & 220 & -21.69 & Em \\
\hline $16358+6709 b$ & 15.0 & BAO $2.6 \mathrm{~m}$ & 0.0537 & 15687 & 221 & -21.70 & HII \\
\hline $16365+6403 a$ & 15.7 & $\mathrm{SAO} 6 \mathrm{~m}$ & 0.0628 & 18250 & 257 & -21.35 & HII \\
\hline $16365+6403 b$ & 17.5 & $\mathrm{SAO} 6 \mathrm{~m}$ & 0.0628 & 18250 & 257 & -19.55 & HII \\
\hline $16365+6403 c$ & 12.6 & $\mathrm{SAO} 6 \mathrm{~m}$ & 0.0632 & 18362 & 259 & -24.46 & HII \\
\hline $16452+6418 a$ & 16.3 & $\mathrm{SAO} 6 \mathrm{~m}$ & 0.0697 & 20183 & 284 & -20.97 & Sy2 \\
\hline $16452+6418 b$ & 16.6 & $\mathrm{SAO} 6 \mathrm{~m}$ & 0.0684 & 19820 & 279 & -20.63 & HII \\
\hline $16452+6418 c$ & 14.2 & $\mathrm{SAO} 6 \mathrm{~m}$ & 0.0685 & 19848 & 280 & -23.03 & HII \\
\hline
\end{tabular}


Table 1 - continued from previous page

\begin{tabular}{|c|c|c|c|c|c|c|c|}
\hline IRAS source & $\mathbf{m}_{V}$ & Spectra source & $\mathbf{z}_{e m}$ & $\mathbf{v}_{r}(\mathrm{~km} / \mathrm{s})$ & $\mathbf{R}(\mathrm{Mpc})$ & $\mathbf{M}$ & Activity type \\
\hline $16452+6418 \mathrm{~d}$ & 17.1 & $\mathrm{SAO} 6 \mathrm{~m}$ & 0.0697 & 20183 & 284 & -20.17 & HII \\
\hline $16533+6216$ & 16.1 & SDSS & 0.1059 & 30089 & 424 & -22.03 & HII \\
\hline $16588+6357$ & 15.6 & SDSS & 0.0604 & 17568 & 247 & -21.40 & Em \\
\hline $17008+6444$ & 15.0 & SDSS & 0.0273 & 8076 & 114 & -20.26 & HII \\
\hline $17008+6444$ & 12.6 & $\mathrm{SAO} 6 \mathrm{~m}$ & 0.0278 & 8224 & 116 & -22.72 & HII \\
\hline $17017+6416$ & 15.2 & SDSS & 0.0843 & 24238 & 341 & -22.43 & HII \\
\hline $17017+6416$ & 14.1 & $\mathrm{SAO} 6 \mathrm{~m}$ & 0.0848 & 24365 & 343 & -23.58 & HII \\
\hline $17037+6207$ & 17.2 & SDSS & 0.1558 & 43138 & 608 & -21.73 & Em \\
\hline $17037+6207$ & 15.8 & SDSS & 0.0823 & 23669 & 333 & -21.86 & HII \\
\hline $17046+6255$ & 16.4 & SDSS & 0.0883 & 25318 & 357 & -21.39 & HII \\
\hline $17046+6255$ & 20.6 & SDSS & 0.0874 & 25086 & 353 & -17.17 & HII \\
\hline $17062+7544$ & 16.1 & BAO $2.6 \mathrm{~m}$ & 0.0654 & 18974 & 267 & -21.02 & Em \\
\hline $17089+6558$ & 17.3 & SDSS & 0.0278 & 8238 & 116 & -18.04 & HII/LINER \\
\hline $17089+6558 a$ & 17.6 & BAO $2.6 \mathrm{~m}$ & 0.0279 & 8256 & 116 & -17.73 & HII \\
\hline $17089+6558 b$ & 15.6 & BAO $2.6 \mathrm{~m}$ & 0.0282 & 8335 & 117 & -19.75 & HII \\
\hline $17102+6442$ & 16.1 & SDSS & 0.0789 & 22735 & 320 & -21.42 & HII \\
\hline $17102+6442$ & 14.4 & $\mathrm{SAO} 6 \mathrm{~m}$ & 0.0789 & 22739 & 320 & -23.13 & HII \\
\hline $17173+6119$ & 15.7 & SDSS & 0.0717 & 20732 & 292 & -21.67 & HII \\
\hline $17173+6119$ & 14.7 & $\mathrm{SAO} 6 \mathrm{~m}$ & 0.0735 & 21242 & 299 & -22.68 & Composite \\
\hline $17190+6219$ & 16.7 & SDSS & 0.0803 & 23117 & 326 & -20.84 & AGN \\
\hline $17190+6219$ & 17.0 & $\mathrm{SAO} 6 \mathrm{~m}$ & 0.0808 & 23264 & 328 & -20.58 & Norm. \\
\hline $17207+6307$ & 15.4 & SDSS & 0.0338 & 9972 & 140 & -20.30 & HII \\
\hline $17207+6307$ & 15.3 & $\mathrm{SAO} 6 \mathrm{~m}$ & 0.0336 & 9911 & 140 & -20.42 & HII \\
\hline $17330+7619$ & 17.2 & $\mathrm{BAO} 2.6 \mathrm{~m}$ & 0.0769 & 22185 & 312 & -20.26 & HII \\
\hline $17349+6139 a$ & 16.2 & $\mathrm{SAO} 6 \mathrm{~m}$ & 0.0858 & 24639 & 347 & -21.50 & HII \\
\hline $17349+6139 b$ & 18.8 & $\mathrm{SAO} 6 \mathrm{~m}$ & 0.0862 & 24749 & 349 & -18.91 & Norm. \\
\hline $17442+6130$ & 14.0 & $\mathrm{SAO} 6 \mathrm{~m}$ & 0.0365 & 10750 & 151 & -21.90 & Norm. \\
\hline $17469+6416$ & 14.6 & $\mathrm{SAO} 6 \mathrm{~m}$ & 0.0355 & 10461 & 147 & -21.24 & HII \\
\hline $17552+6209$ & 16.4 & $\mathrm{SAO} 6 \mathrm{~m}$ & 0.0836 & 24035 & 339 & -21.25 & Sy2 \\
\hline $17591+8628$ & 15.2 & BAO $2.6 \mathrm{~m}$ & 0.0233 & 6917 & 97 & -19.72 & HII \\
\hline $18116+6328$ & 13.9 & $\mathrm{SAO} 6 \mathrm{~m}$ & 0.0481 & 14083 & 198 & -22.59 & Norm. \\
\hline $18169+6433 \mathrm{c}$ & 12.4 & $\mathrm{SAO} 6 \mathrm{~m}$ & 0.0208 & 6175 & 87 & -22.30 & HII \\
\hline $18169+6433 d$ & 15.1 & $\mathrm{SAO} 6 \mathrm{~m}$ & 0.0209 & 6204 & 87 & -19.61 & HII \\
\hline $18192+8650$ & 16.3 & BAO $2.6 \mathrm{~m}$ & 0.0655 & 19002 & 268 & -20.88 & HII \\
\hline $18247+6102$ & 15.7 & $\mathrm{SAO} 6 \mathrm{~m}$ & 0.0732 & 21158 & 298 & -21.67 & LINER \\
\hline $18252+6315$ & 15.9 & $\mathrm{SAO} 6 \mathrm{~m}$ & 0.0838 & 24090 & 339 & -21.75 & HII \\
\hline $18380+8640 b$ & 16.1 & BAO $2.6 \mathrm{~m}$ & 0.0778 & 22435 & 316 & -21.43 & HII \\
\hline $18380+8640 c$ & 16.0 & BAO $2.6 \mathrm{~m}$ & 0.0779 & 22451 & 316 & -21.46 & HII \\
\hline $20537+8737$ & 14.8 & BAO $2.6 \mathrm{~m}$ & 0.0195 & 5784 & 81 & -19.77 & Em \\
\hline
\end{tabular}

The objects have redshifts in the range $0.0092<z<0.1997$ and absolute stellar magnitudes in the range $-16.81<M<-24.46$. The distribution of redshifts is given in Fig. 1 and the distribution of absolute magnitudes is given in Fig. 2.

\section{Summary and conclusion}

Spectral observations of BIG objects at the BAO 2.6-m, SAO 6-m, OHP 1.93-m telescopes and taken from SDSS database have yielded a fairly rich set of data for studying IR galaxies. These data can be used to study the BIG sample, and also to compare these objects with IR galaxies from other samples. A total of 257 BIG objects were observed; of these 149 were identified as galaxies with star formation regions, 42 as AGN, 28 as galaxies with a composite spectrum (referred below 


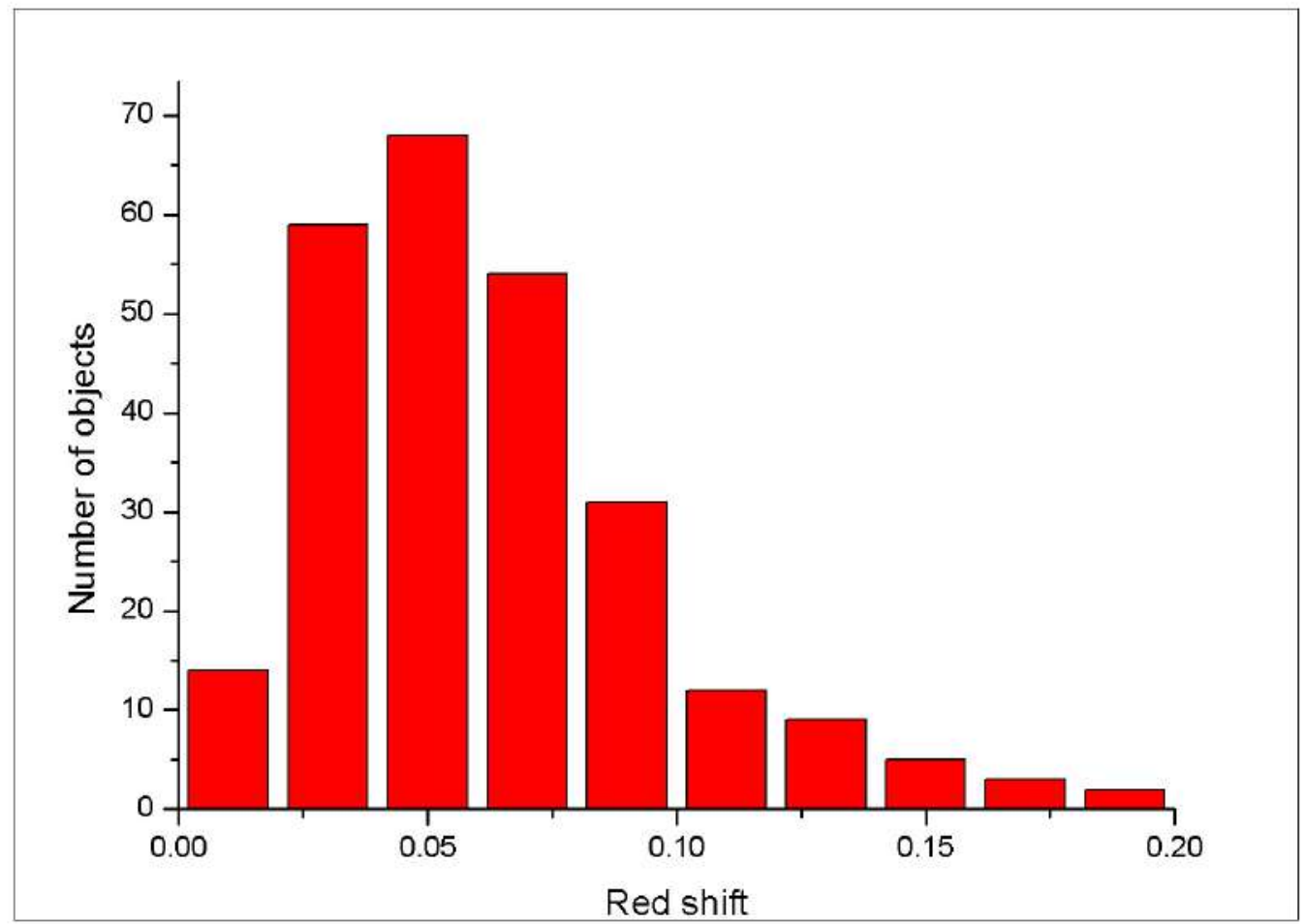

Figure 1. Distribution of redshifts

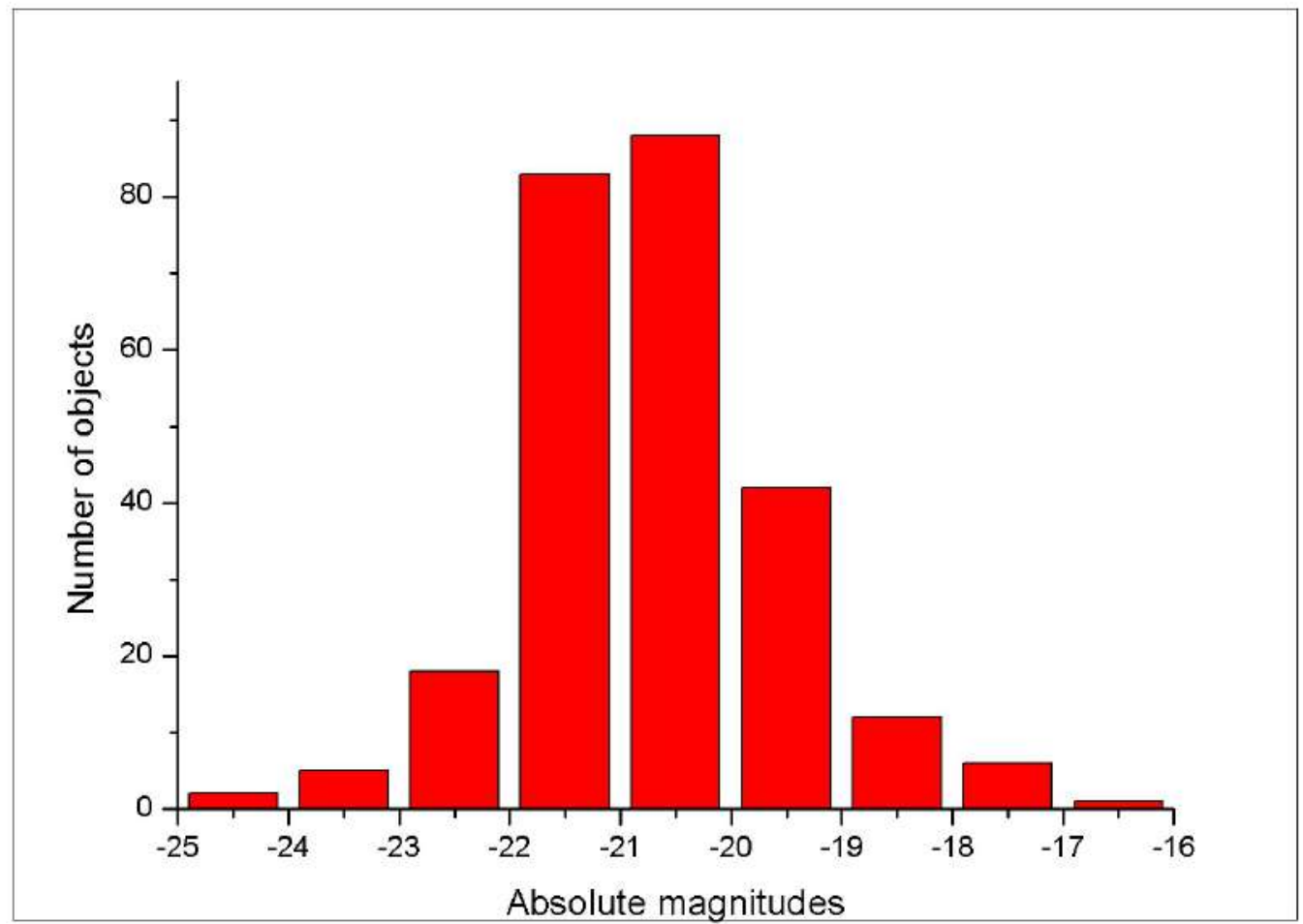

Figure 2. Distribution of absolute magnitudes

to Composite or Comp), 21 as "Em" galaxies (this type refers to a spectrum with signs of emission without the possibility of a more precise determination of the activity class of the galaxy), 13 as galaxies represented as Norm (galaxies for which the rate of star formation does not exceed the normal), 3 as 
absorption galaxy and one object without the possibility of classification (unknown). In Table 2 the distribution of all objects by activity types is given.In Fig. 3 absolute magnitude $(M)$ vs redshift $(z)$ is given.

Table 2: The distribution of 257 BIG objects by activity types

\begin{tabular}{|c|c|c|c|c|c|}
\hline Activity type & Number of objects & $\%$ & Activity type & Number of objects & $\%$ \\
\hline HII & 149 & 58.0 & AGN & 13 & 5.1 \\
Composite & 22 & 8.6 & Em & 21 & 8.2 \\
HII / LINER & 4 & 1.5 & Norm & 13 & 5.1 \\
HII / Sy & 2 & 0.8 & Abs & 3 & 1.1 \\
LINER & 12 & 4.6 & Unknown & 1 & 0.4 \\
Sy & 17 & 6.6 & All & $\mathbf{2 5 7}$ & $\mathbf{1 0 0 . 0}$ \\
\hline
\end{tabular}

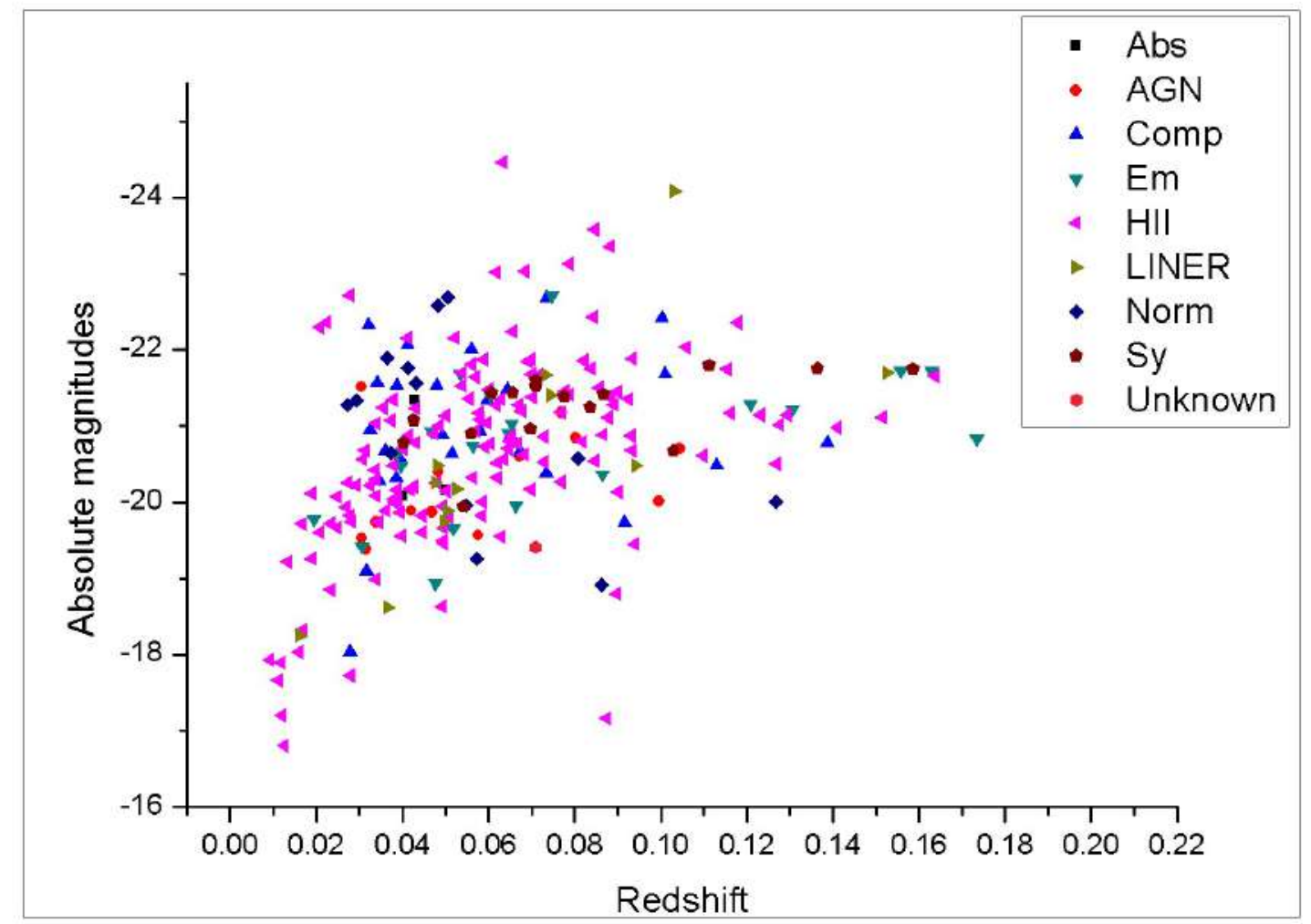

Figure 3. The distribution of absolute magnitudes vs redshifts

Besides isolated galaxies, the observed objects include some binary and multiple systems. This makes it possible to establish their physical coupling, to determine the true IR source more precisely (as an individual galaxy or the system as a whole), and to study the interrelation between star formation activity, the interactions of galaxies, and the activity of their nuclei.

\section{References}

M. Moshir, G. Kopan, T. Conrow, and et al. Infrared Astronomical Satellite Catalogs, The Faint Source Catalog, Version 2.0, 1990 .

H.V. Abrahamyan, A.M. Mickaelian, and A.V. Knyazyan. Astron. Comput. 10, 99, 2015.

D.B. Sanders and I.F. Mirabel. Luminous Infrared Galaxies, Ann. Rev. Astron. Astrophys. 34, 749, 1996.

R.M. Cutri, M.F. Skrutskie, S. van Dyk, and et al. 2MASS All-Sky Catalog, Univ. Mass. and IPAC/CalTech, 2003. 
M.F. Skrutskie, R.M. Cutri, R. Stiening, M.D. Weinberg, S. Schneider, J.M. Carpenter, C. Beichman, R. Capps, and et al. Astron. J. 131, 1163, 2006.

R.M. Cutri, E.L. Wright, T. Conrow, and et al. WISE All-Sky DR, IPAC/Caltech, 2013.

D. Ishihara, T. Onaka, H. Kataza, A. Salama, C. Alfageme, A. Cassatella, N. Cox, P.García-Lario, and et al. Astron. Astrophys. $514,1,2010$.

I. Yamamura, S. Makiuti, N. Ikeda, Y. Fukuda, S. Oyabu, T. Koga, and G.J. White. AKARI/FIS, ISAS/JAXA, 2010.

D.B. Sanders, J.M. Mazzarella, D.-C. Kim, J.A. Surace, and B. T. Soifer. Astron. J. 126, 1607, 2003.

E. Bertin, M. Dennefeld, and M. Moshir. Astron. Astrophys. 323, 685, 1997.

M.A. Strauss and J.P. Huchra. Astron. J. 95, 1602, 1988.

G. Wang, S.K. Leggett, R.G. Clowes, H.T. MacGillivray, and A. Savage. MNRAS 248, 112, 1991.

K.B. Fisher, J.P. Huchra, M.A. Strauss, M. Davis, A. Yahil, and D. Schlegel. Astrophys. J. Suppl. Ser. 100, 69, 1995

Q.R. Yuan, Z.H. Zhu, Z.L. Yang, and X.T. He. Astron. Astrophys. Suppl. Ser. 115, 267, 1996.

A.M. Mickaelian. Astrophysics 38, 625, 1995.

D.W. Weedman, F.R. Feldman, V.A. Balzano, and et al. Astrophys. J. 248, 105, 1981.

V.A. Ambartsumian. XI Solvay Conference, Editions Stoops, Brussels, 1958.

A.M. Mickaelian and L.A. Sargsyan. Astrofizika 47, 109, 2004.

A.M. Mickaelian and K.S. Gigoyan. Astron. Astrophys. 455, 765. Catalog No. III/237a in Vizier, CDS, Strasbourg, 2006.

B.E. Markarian, V.A. Lipovetsky, J.A. Stepanian, L.K. Erastova, and A.I. Shapovalova. Comm. SAO 62, 5, 1989.

A.M. Mickaelian, R. Nesci, C. Rossi, D. Weedman, G. Cirimele, L.A. Sargsyan, L.K. Erastova, K.S. Gigoyan, and et al. Astron. Astrophys. 464, 1177, 2007.

E. Massaro, A.M. Mickaelian, R. Nesci, and D. Weedman. The Digitized First Byurakan Survey (Roma, Italy), 2008.

A.M. Mickaelian, L.R. Oganesyan, and L.A. Sargsyan. Astrofizika 46, 221, 2003.

L.A. Sargsyan and A.M. Mickaelian. Astrofizika 49, 19, 2006.

A.M. Mickaelian, S.A. Akopyan, S.K. Balayan, and A.N. Burenkov. Pis'ma v Astron. zh. 24, 736, 1998.

S.K. Balayan, S.A. Akopyan, A.M. Mickaelian, and A.N. Burenkov. Pis'ma v Astron. zh. 27, 330, 2001.

A.M. Mickaelian. Astrofizika 47, 425, 2004.

B. Abolfathi, D.S. Aguado, G. Aguilar, and et al. The Astrophysical Journal Supplement Series, 235, issue 2, article id. 42, p. 19, 2018.

A.M. Mickaelian, G.S. Harutyunyan, and A. Sarkissian. Astronomy Letters, Volume 44, Issue 6, pp.351-361, 2018.

Joint IRAS Science Working Group. Infrared Astronomical Satellite Catalogs, The Point Source Catalog, Version 2.0, NASA $R P-1190,1988$ 